\title{
NONINVASIVE MONITORING OF RADIOTHERAPY-INDUCED MICROVASCULAR CHANGES USING DYNAMIC CONTRAST ENHANCED MAGNETIC RESONANCE IMAGING (DCE-MRI) IN A COLORECTAL TUMOR MODEL
}

\author{
Wim Ceelen, M.D., ${ }^{*}$ Peter Smeets, M.D., ${ }^{\dagger}$ Walter Backes, Ph.D. ${ }^{\ddagger}$ Nancy Van Damme, Ph.D., ${ }^{\S}$ \\ Tom Boterberg, M.D., Ph.D." Pieter Demetter, M.D., Ph.D., II Isabel Bouckenooghe,* \\ Marieke De Visschere, ${ }^{*}$ Marc Peeters, M.D., Ph.D., ${ }^{\S}$ and Piet Pattyn, M.D., Ph.D.*
}

Departments of *Surgery, ${ }^{\dagger}$ Radiology, ${ }^{\S}$ Gastroenterology, and ${ }^{\|}$Radiotherapy, Ghent University Hospital, Ghent, Belgium;

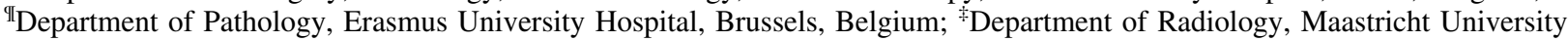
Hospital, Maastricht, The Netherlands

\begin{abstract}
Purpose: To examine dynamic contrast-enhanced magnetic resonance imaging (DCE-MRI) with a macromolecular contrast agent (P792) to visualize effects of radiotherapy (RT) on microvascular leakage in a colorectal cancer model.

Methods and Materials: CC531 tumors were induced in WAG/Rij rats. DCE-MRI was performed before and 5 days after $5 \times 5$ Gy of RT and parametric maps generated of the endothelial transfer constant $\left(K^{\text {trans }}\right)$ and the fractional interstitial space $\left(V_{e}\right)$ according to the Tofts model. Tissue $\mathrm{pO}_{2}$ mapping was performed in each tumor core and rim before and after RT. Microvessel density (MVD), vascular endothelial growth factor (VEGF) expression, and pimonidazole hypoxia staining were compared with a control group of tumor-bearing rats. Results: Mean $K^{\text {trans }}$ and $v_{e}$ were significantly reduced after $\mathrm{RT}$ in all tumor regions. Mean $\mathrm{pO}_{2}$ was $6.8 \mathrm{~mm} \mathrm{Hg}$ before RT vs. $7.7 \mathrm{~mm} \mathrm{Hg}$ after RT $(p<0.001)$ in the tumor rim and $3.5 \mathrm{~mm} \mathrm{Hg}$ before RT vs. $4.4 \mathrm{~mm} \mathrm{Hg}$ after RT $(p<0.001)$ in the tumor core. Mean MVD in the tumor rim was 10.4 in the RT treated group vs. 16.9 in the control group $(p=0.061)$. VEGF expression was significantly higher in RT-treated rats. After RT, no correlation was found between DCE-MRI parameters and histologic parameters. A correlation was seen after RT between $\mathrm{pO}_{2}$ and $K^{\text {trans }}(r=-0.57, p=0.08)$ and between $\mathrm{pO}_{2}$ and $v_{e}(r=-0.65, p=0.04)$.

Conclusions: Dynamic contrast-enhanced-MRI with P792 allows quantification of microvascular changes in this colorectal model. RT significantly reduces neovascular leakage and enhances tissue oxygenation and VEGF expression. After RT, DCE-MRI parameters are related to tumor $\mathrm{pO}_{2}$, but not to MVD or VEGF expression. (C) 2006 Elsevier Inc.
\end{abstract}

Colorectal, Magnetic resonance, DCE-MRI, Radiotherapy.

\section{INTRODUCTION}

Colorectal cancer remains one of the leading causes of cancer death in the Western world (1). Because of the specific anatomy and biology of low rectal cancer, surgical resection alone historically has been associated with a high incidence of locally recurrent disease. Neoadjuvant combined modality treatment has been shown to decrease local recurrence rates even with the use of optimal surgical technique (total mesorectal excision) $(2,3)$. Potential disadvantages of neoadjuvant chemoradiation include overtreatment of overstaged disease, treatment-related toxicity, and long-term effects on sphincter function (4). There is therefore a need for noninvasive imaging techniques that allow clinicians to predict and monitor tumor response to neoadjuvant therapy.

Magnetic resonance imaging (MRI) recently has evolved as one of the most promising imaging modalities in the diagnosis and staging of rectal cancer (5). Dynamic contrast-enhanced magnetic resonance imaging (DCE-MRI) using paramagnetic contrast agent administration allows one to analyze tumor specific enhancement patterns that are governed by physiologic properties such as microvascular flow, endothelial permeability, and size of the extracellular extravascular space (EES) (6). Quantitative analysis of the enhancement signal using a pharmacokinetic model can gen-
Reprint requests to: W. Ceelen, M.D., Department of Surgery 2K12 IC, Ghent University Hospital, De Pintelaan 185, B-9000 Ghent, Belgium. Tel: (+32) 9-240 62 51; Fax: (+32) 9-240 38 91; E-mail: Wim.ceelen@ugent.be

Acknowledgments-The CC531 cells were kindly provided by the Laboratory of Cell Biology and Histology, Free University of Brus- sels, Belgium. Gadomelitol (P792) was kindly provided by Guerbet (Roissy, France), who did not provide any other financial or material support to this study. We thank Louis Cesteleyn and Philippe Houtmeyers for their technical assistance during the experiments.

Received Mar 17, 2005, and in revised form Oct 12, 2005. Accepted for publication Oct 29, 2005. 
erate estimates for parameters such as the endothelial transfer coefficient $K^{\text {trans }}$ (7). The MRI contrast agents in clinical use have a low $(<1 \mathrm{kD})$ molecular weight and diffuse readily across the endothelial barrier of both normal and neoplastic tissues and therefore are much less suited to characterize hyperpermeable neoplastic vessels (8). Macromolecular weight contrast media take advantage of the selective hyperpermeability of neoplastic vessels to macromolecules and have been used successfully to monitor angiogenesis and the effects of antiangiogenesis agents in preclinical studies (9). For macromolecular agents, microvascular leakage determined by $K^{\text {trans }}$ is relatively flow independent and therefore estimation of leakage changes as a surrogate marker of angiogenesis is possible without exact measurement of capillary flow (10).

We studied the changes in neovascular leakage of an experimental colorectal cancer during fractionated radiotherapy (RT) using DCE-MRI with P792, a new macromolecular MRI contrast agent (CA) evaluated in phase II clinical trials. Noninvasive imaging was compared with invasive tissue $\mathrm{pO}_{2}$ measurement, microvessel counts, vascular endothelial growth factor (VEGF) expression, and pimonidazole hypoxia staining.

\section{METHODS AND MATERIALS}

The experimental protocol was reviewed and approved by the Animal Ethical Committee of the Ghent University, Belgium.

\section{Animals and tumor model}

A group of 11 male Wag/Rij rats (Harlan, Horst, The Netherlands) was studied longitudinally with DCE-MRI and invasive oxygenation measurements performed both before and 5 days after completion of RT. For histology and immunohistochemistry, this group was compared with nine untreated control rats bearing tumors of similar size. The CC531 cell line is a 1,2-dimethylhydrazine-induced, moderately differentiated, and weakly immunogenic colon adenocarcinoma, syngeneic with WAG/Rij rats. This cell line is well studied and has been proven to provide a tumorhost model similar to human colorectal carcinogenesis (11). Cells were grown as a stationary cell line in plastic culture flasks in RPMI 1640 medium, buffered with HEPES $(20 \mathrm{mM})$ (Invitrogen Corporation, Gibco, Ghent, Belgium) additionally supplemented with $10 \%$ fetal calf serum, $4 \mathrm{mM}$ L-glutamine, $50 \mathrm{U} / \mathrm{mL}$ penicillin, and $50 \mu \mathrm{g} / \mathrm{mL}$ streptomycin at $37^{\circ} \mathrm{C}$ in a humidified atmosphere with $5 \% \mathrm{CO}_{2}$ in the air. The cells were transferred at $95 \%$ confluency. Two million cells suspended in $0.2 \mathrm{~mL}$ were injected subcutaneously in the upper hind leg. Tumors reached a size of $0.5-1 \mathrm{~cm}$ after a period of 4 weeks. After tumor growth of minimally $8 \mathrm{~mm}$ diameter was observed, a jugular vein catheter was inserted and tunneled to the interscapular region. To maintain catheter delivery function between the first and second MRI, continuous infusion at $0.5 \mathrm{~mL}$ saline/h was administered with a cage-mounted swivel and flexible metal tether system (Uno BV, Didam, The Netherlands), allowing the animal full mobility. To enable histologic assessment, animals were sacrificed by anesthetic overdose after the last in vivo measurements.

\section{Radiotherapy}

Rats were not sedated and the tumor-bearing hind leg was immobilized using a Plexiglas holder, as described previously $(12,13)$.
Briefly, rats were placed in a purpose-built Plexiglas holder in prone position. The hind legs were pulled through an opening in the holder and immobilized with strings. Before each fraction, a radiation field was simulated encompassing the tumor with a margin of $1.5 \mathrm{~cm}$. The photon irradiation was performed with a $5 \mathrm{MV}$ linear accelerator (Elekta, Crawley, UK). Five fractions of 5 Gy (total dose, $25 \mathrm{~Gy}$ ) were delivered on 5 consecutive days. Because the tumors were inoculated subcutaneously, they were covered with tissue-equivalent silicone bolus of $1 \mathrm{~cm}$ to prevent the buildup effect under the skin. One single direct field at a fixed source-skin distance of $100 \mathrm{~cm}$ was used. The dose was calculated to the midpoint of the tumors according to their volume in each individual animal, as obtained during simulation. DCE-MRI and oxygenation measurements were performed before and 5 days after the completion of RT.

\section{$M R I$}

$\mathrm{T}_{1}$ weighted DCE-MRI was performed on a clinical Siemens Magnetom Symphony 1.5 Tesla scanner (Siemens AG, Erlangen, Germany). Animals were sedated with $0.2-0.4 \mathrm{~mL}$ of medetomidine (Domitor, Novartis Animal Health, Basel, Switzerland). Imaging comprised a single axial slice that was positioned through both upper limbs and the center of the tumor. Before the contrast series, $\mathrm{T}_{1}$ zero time maps were constructed from two spin echo sequences with different repetition times (repetition time, $1000 \mathrm{~ms}$ and $318 \mathrm{~ms}$, respectively). Details of this sequence were as follows: slice thickness $3 \mathrm{~mm}$, field of view $140 \times 88$, matrix size $256 \times 160$, echo time $20 \mathrm{~ms}$, and flip angle $90^{\circ}$. Dynamic imaging was performed with a four-antenna wrist coil (diameter, $10 \mathrm{~cm}$ ) using an IR-TurboFLASH sequence. Details of the pulse sequence were as follows: temporal resolution $1.1 \mathrm{~s}$ (i.e., repetition time, $1100 \mathrm{~ms}$ ), field of view $140 \times 88$, matrix size $256 \times 160$, slice thickness $5 \mathrm{~mm}$, echo time $4.08 \mathrm{~ms}$, inversion time $560 \mathrm{~ms}$, and flip angle: $12^{\circ}$. A bolus injection of $35-50 \mu \mathrm{M} \mathrm{Gd} / \mathrm{Kg}$ of P792 was manually injected as fast as possible (approximately $1 \mathrm{~mL} / \mathrm{s}$ ) through the central venous line after the fourth scan. A total of 500 images were obtained for a total scan time of $550 \mathrm{~s}$.

\section{Analysis of MR images}

Postprocessing was performed using the research mode of a commercially available software tool (MIStar, Apollo Medical Imaging, Melbourne, Australia).

The maximum cross sectional area (in $\mathrm{cm}^{2}$ ) of each tumor was recorded before and after RT.

Both a qualitative description of the tissue enhancement curve and a two-compartment pharmacokinetic approach according to Tofts and Kermode were implemented (7). In each tumor, regions of interest were drawn encompassing the following regions: (1) the entire tumor, (2) the angiogenic tumor rim defined as the outer 2-3 voxel wide circumference, (3) the central necrotic area, and (4) muscle tissue in the contralateral non irradiated hind leg.

In each region, the area under the enhancement curve (AUC) was calculated until $550 \mathrm{~s}$ after contrast arrival.

Pharmacokinetic modeling was based on a two-compartment model consisting of a vascular space and an EES fraction that is entered by the CA leaking through the microvascular wall. Derived from a first-order differential equation describing contrast agent flux driven by Fick's law of diffusion, the concentration of CA in the tissue $\left(C_{t}\right.$ in $\left.\mathrm{mM}\right)$ is described by the following equation:

$$
C_{t}=K^{\text {trans }} \int_{0}^{t} C_{p}\left(t^{\prime}\right) e^{\frac{-K^{\text {rrans }}}{v_{e}}\left(t-t^{\prime}\right)} d t^{\prime}
$$


where $K^{\text {trans }}\left(\min ^{-1}\right)$ denotes the endothelial transfer rate, $v_{e}$ the fraction of the interstitial space (dimensionless) entered by the CA, and $C_{p}$ the plasma concentration (in $\mathrm{mM}$ ). In each tumor both before and after RT, the pixel with the most representative arterial input function was manually selected from the femoral artery of the tumor bearing leg or the opposite leg to provide $C_{p}(t)$. Mean tumor tissue and arterial $\mathrm{T}_{1}$ zero (before contrast injection) values were calculated from the $T_{1}$ zero maps. These data, together with the selected arterial input function, were used as the input for a curve fitting routine resulting in parametric maps of $K^{\text {trans }}$ and $v_{e}$. The influence of inflow effects on leakage measurements was minimized by using a deconvolution method in the model to separate the inflow component from the tissue concentration $C_{t}(7)$.

Quantitative values for all pixels in the four different regions of interest described above were exported to a spreadsheet for further analysis.

\section{MR contrast medium}

Dynamic contrast studies were performed with P792 (Gadomelitol, Vistarem, Guerbet, Roissy, France), a new monogadolinated rapid clearance MRI blood-pool agent that is cleared by renal elimination. The molecular weight of the compound is 6.47 $\mathrm{kD}$, but the mean diameter of P792 is 50.5 Angstrom and the T1 relaxivity of this agent is $29 \mathrm{mM}-1 \mathrm{~s}-1$ at $60 \mathrm{MHz}$ (14). Apparent hydrodynamic volume of P792 is 125 times greater than that of Gd-DOTA (gadoterate meglumine, Dotarem); as a result of this high molecular volume, P792 is characterized by a limited diffusion across normal endothelium and is therefore ideally suited to study hyperpermeable neoplastic vessels (14). Experimentally, P792 has been used to study permeability effects of antiangiogenesis therapy in a prostate cancer model (15).

\section{Tissue $\mathrm{pO}_{2}$ measurements}

Tissue oxygenation was measured in both the tumor core and periphery with a fiberoptic probe based on fluorescence quenching (OxyLite, Oxford Optronix, Oxford, UK) $(16,17)$. A precalibrated fiberoptic probe (diameter, $280 \mu \mathrm{m}$ ) was inserted $5 \mathrm{~mm}$ deep into the tumor using a Seldinger technique. This involved insertion of a catheter with needle assembly into the tissue; the needle was then withdrawn leaving the catheter in place, through which the probe was inserted in such a way that the probe's tip was exposed to the surrounding tissue. The probe was then withdrawn in 40 steps of 100 $\mu \mathrm{m}$ each over a total distance of $4 \mathrm{~mm}$ using a micromanipulator (model MN151, Narishige International Ltd, London, UK). After each micromanipulator movement, measurements were started as soon as a stable reading was obtained. Tissue $\mathrm{pO}_{2}$ was sampled every $2 \mathrm{~s}$. Histograms were constructed based on the $\mathrm{pO}_{2}$ (expressed in $\mathrm{mm}$ $\mathrm{Hg}$ ) readings over the central $1 \mathrm{~mm}$ (core) and outer $1 \mathrm{~mm}$ (periphery) trajectory. Both before and after RT, the hypoxic fraction (defined as percentage of $\mathrm{pO}_{2}$ measurements with a value of $<5 \mathrm{~mm} \mathrm{Hg}$ ) was determined.

\section{Immunohistochemistry}

Immunohistochemistry was performed on the experimental group and on nine untreated control rats bearing tumors of similar size. From each tumor, half of the tissue was snap frozen in liquid nitrogen; the other part was fixed in $4 \%$ formalin and embedded in paraffin wax in the conventional manner. Microvascular density (MVD) was determined with a method modified after Weidner et al. (18) After incubating $5 \mu \mathrm{m}$ frozen slices with anti-CD31 antibodies (mouse anti rat CD31, clone number TLD-3A12, Serotec, Oxford, UK), the entire tumor section was scanned at low power (objective, $40 \times$ ) to identify "hot spots," which are the areas of highest neovascularization. Individual microvessels were then counted under higher power (objective, $400 \times$ ) to obtain a vessel count in a defined area, and the average vessel count in three hot spots was taken as the MVD.

For hypoxia staining, rats were injected with $60 \mathrm{mg} / \mathrm{kg}$ intravenous pimonidazole (Hydroxyprobe Kit, Biognost, Heule, Belgium) 30 min before sacrifice. Paraffin-embedded slides were incubated with antipimonidazole antibodies and the resulting cytoplasmic staining was expressed semiquantitatively. Membrane staining was not observed. Immunoassaying was visualized using 3-amino-9ethylcarbazole (Dako, Glostrup, Denmark).

VEGF expression was assessed after incubating slides with mouse anti-human VEGF C1 monoclonal antibody (sc-7269, Santa Cruz Biotechnology Inc., Santa Cruz, CA). The antibody used reacts with VEGF of mouse, rat, and human origin. Semiquantitative scoring of both pimonidazole staining and VEGF expression was based on a method modified after Coppola et al. (19), with a scale ranging from 0 to 6 . The scale was based on scoring of the fraction of positive cells (0: all cells negative; $1:<33 \%$ positive; $2: 33-66 \%$ positive; 3 : $>66 \%$ positive) and the staining intensity (1: weak; 2 : moderate; 3 : intense). Both scores were added to a maximum score of 6 .

\section{Statistical analysis}

Data were analyzed and presented graphically with a statistical software package (S-PLUS 6.1 for Windows, Insightful Corporation,

\section{(a)}

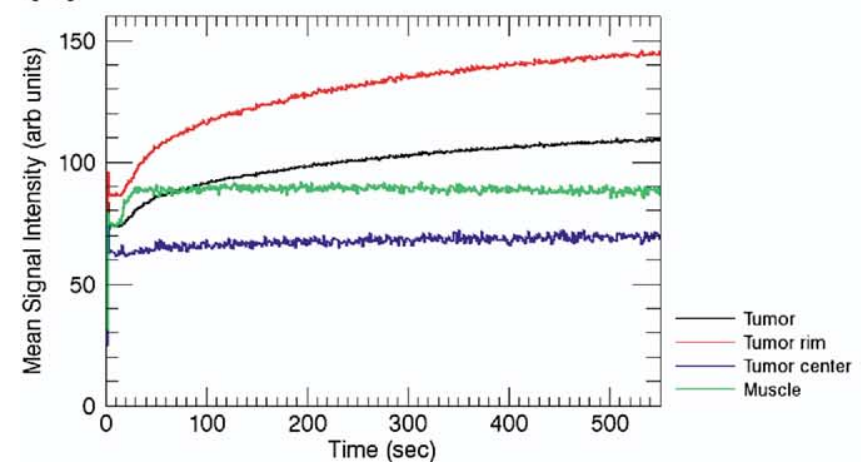

(b)

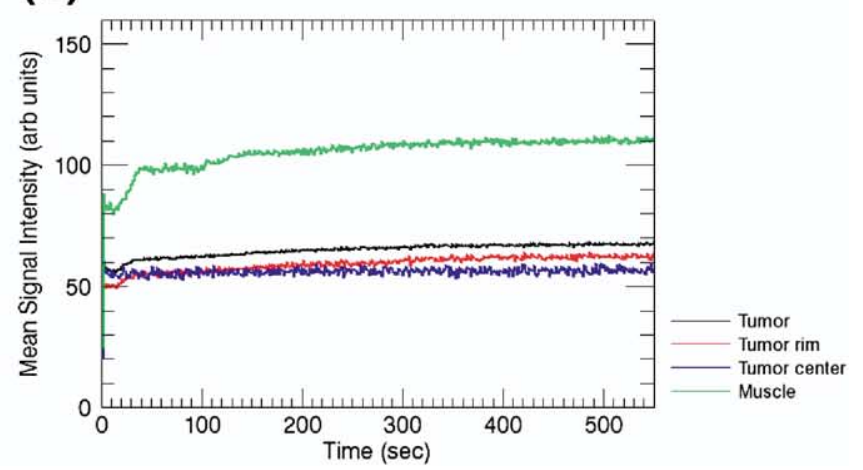

Fig. 1. Typical enhancement pattern in different colorectal tumor regions and normal muscle before (a) and after (b) fractionated radiotherapy. Curves represent changes in signal enhancement in a single tumor. 
Seattle, WA). Differences in signal enhancement parameters between four tissue regions were evaluated with one-way analysis of variance. Differences in imaging parameters and $\mathrm{pO}_{2}$ before vs. after RT were assessed with the paired-sample $t$ test or, when data were not normally distributed, with the Wilcoxon signed-rank test. Differences in MVD between RT-treated rats and control rats were analyzed with the unpaired $t$ test, whereas differences in pimonidazole staining score and VEGF expression were analyzed with the Mann-Whitney U test. Correlation analyses between imaging, oxygenation, and histologic data were performed with the Spearman rank order test. Statistical significance was inferred when $p \leq 0.05$. Data are expressed as mean (95\% confidence interval) unless stated otherwise.

\section{RESULTS}

\section{Effect of RT on tumor growth}

The mean cross-sectional surface area of the tumor did not change significantly during RT: $1.5(0.9-2.1) \mathrm{cm}^{2}$ before RT and $1.6(0.8-2.4) \mathrm{cm}^{2}$ after RT $(p=0.9)$.

\section{Enhancement of neoplastic vs. normal tissue}

A similar temporal enhancement pattern was observed in all animals (Fig. 1) confirming selective MRI enhancement of tumor tissue by P792. Before RT, the arterial input (a)

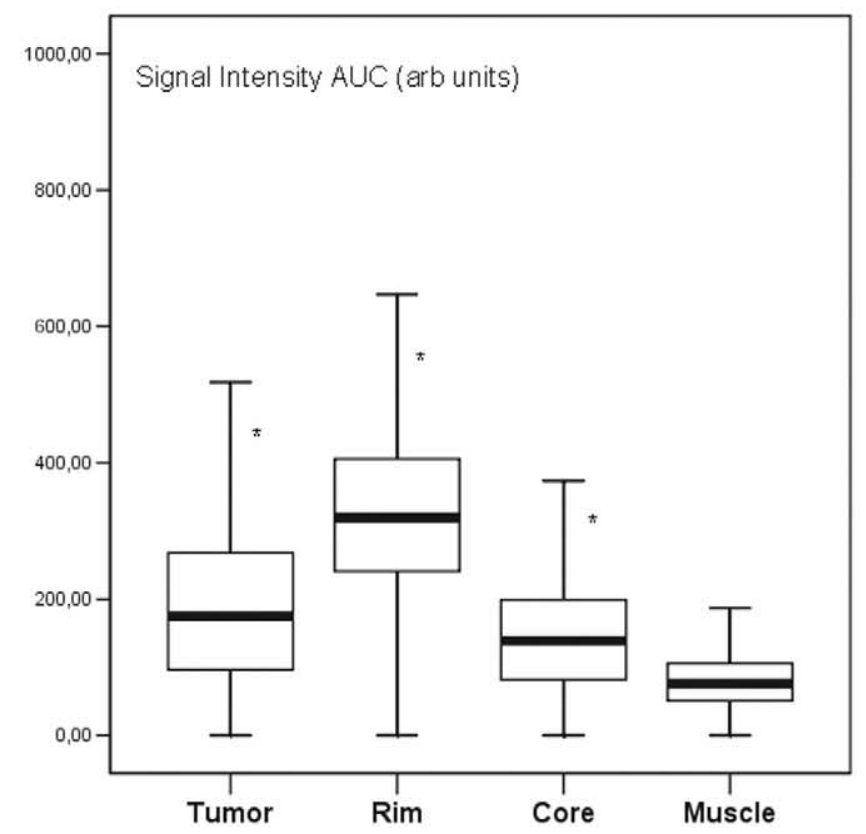

(b)

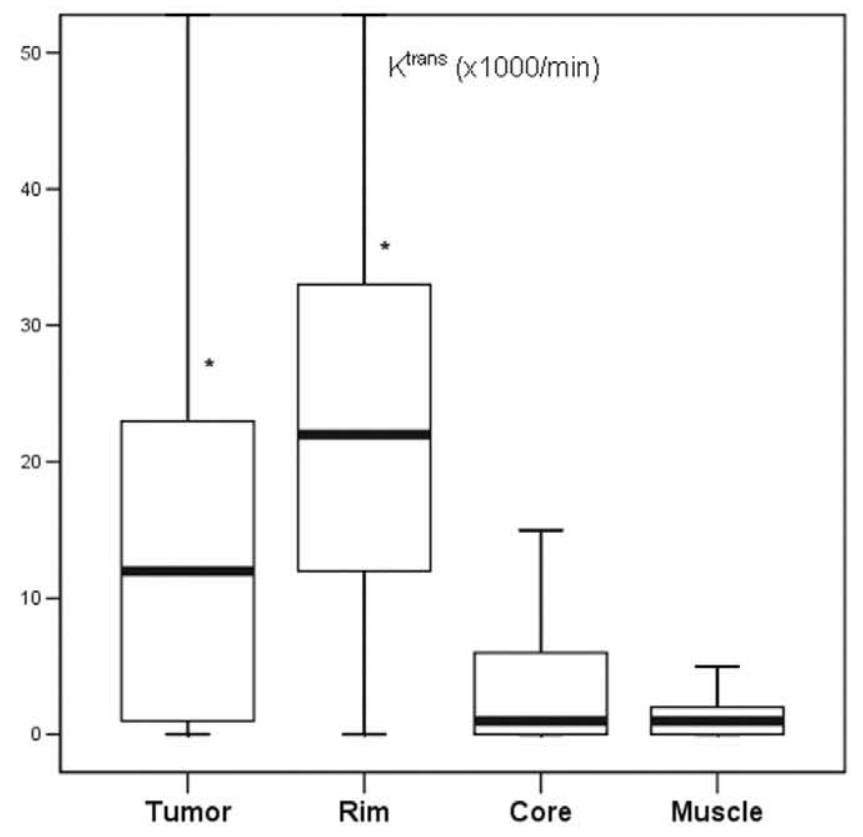

(c)



Fig. 2. Cumulative signal intensity and pharmacokinetic parameters before radiotherapy. *Statistically significant compared with normal muscle. AUC = area under the curve. 
(a)

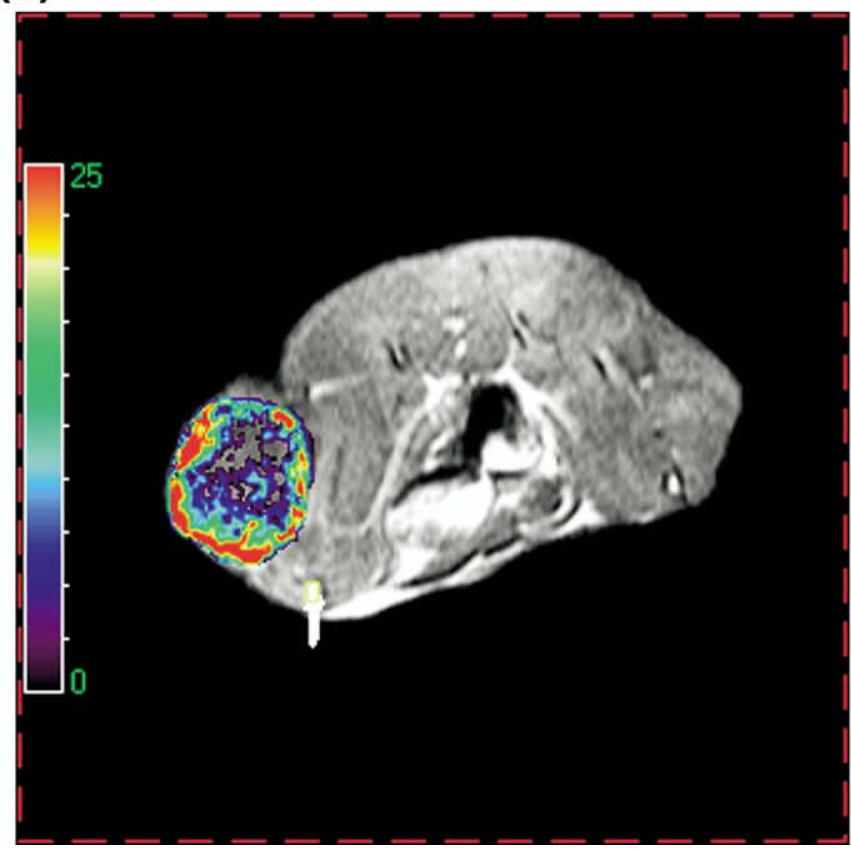

(b)

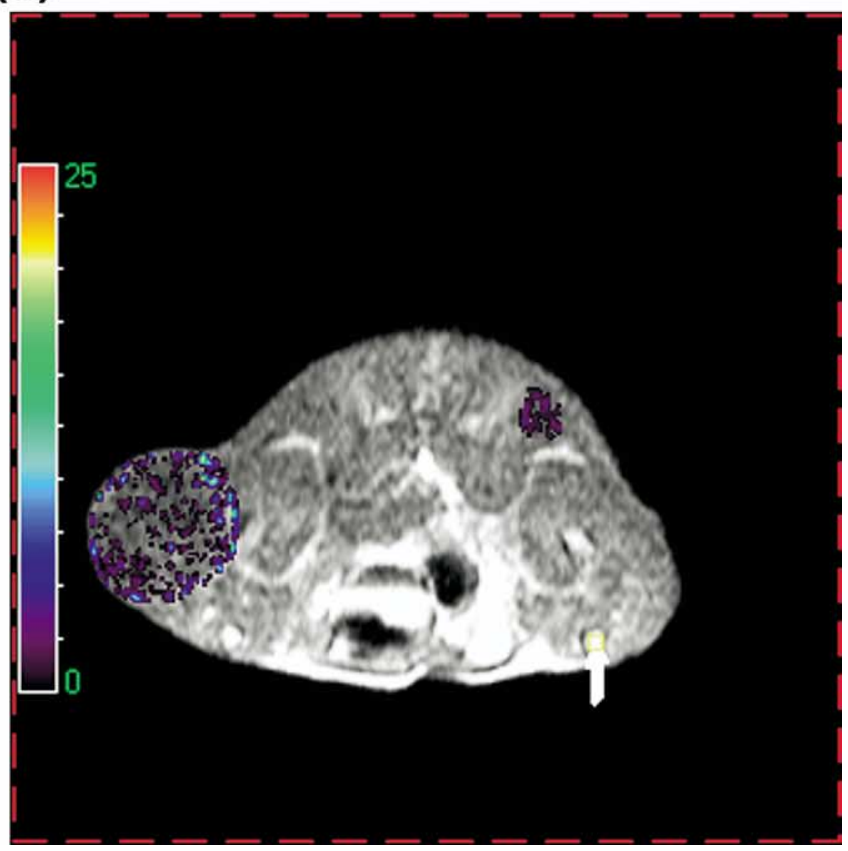

Fig. 3. Parametric map (tumor masked) of $K^{\text {trans }}(\times 1000 / \mathrm{min})$ before (a) and 5 days after (b) $5 \times 5$ Gy of fractionated radiotherapy. The arrow points to the pixel containing the selected arterial input function.

function tended to peak at approximately $100-120$ s. Signal enhancement in neoplastic tissue was characterized by a short initial rapid increase of signal intensity corresponding to the first arterial inflow and initial rapid inwash in the tumor tissue. This 20-25 s phase was followed in tumor tissue by a slowly rising signal intensity increase, which was most pronounced in the tumor rim. The tumor center displayed a similar slow uptake, although the signal intensity was much lower. Normal muscle, however, did not enhance after the initial fast uptake phase.

Cumulative DCE parameter values before RT are illustrated in Fig. 2. Mean AUC of signal intensity was significantly different between entire tumor, tumor rim, tumor core, and normal muscle ( $p<0.001$ for all comparisons). Both pharmacokinetic parameters were significantly different between the entire tumor, tumor rim, and tumor core ( $p<0.001$ for all comparisons), with the highest
$K^{\text {trans }}$ observed at the tumor rim. No significant differences in pharmacokinetic parameters were seen between the tumor core and normal muscle $\left(p=0.4\right.$ for both $K^{\text {trans }}$ and $\left.v_{e}\right)$.

\section{Effects of fractionated RT on pharmacokinetic DCE-MRI parameters}

The effects of fractionated RT were first evaluated graphically with parametric maps of the tumor (Fig. 3a and 3b).

Both $K^{\text {trans }}$ and $v_{e}$ were significantly lower after RT in all three examined regions of the tumor (Table 1). In nonirradiated muscle tissue, on the contrary, no changes were observed in $K^{\text {trans }}$ or $v_{e}$.

\section{Effects of fractionated $\mathrm{RT}$ on tumor tissue $\mathrm{pO}_{2}$}

The mean number of $\mathrm{pO}_{2}$ readings per animal was 839 before RT and 706 after RT. Mean $\mathrm{pO}_{2}$ values in both tumor regions before and after $\mathrm{RT}$ are illustrated in Table 2 and

Table 1. Comparison of pharmacokinetic contrast enhancement parameters before and after radiotherapy (RT) in different regions of interest

\begin{tabular}{|c|c|c|c|c|c|c|}
\hline \multirow[b]{2}{*}{ Parameter } & \multirow{2}{*}{$\begin{array}{c}\text { Region of } \\
\text { interest }\end{array}$} & \multicolumn{2}{|c|}{ Before radiotherapy } & \multicolumn{2}{|c|}{ After radiotherapy } & \multirow[b]{2}{*}{$p$} \\
\hline & & Mean & $95 \% \mathrm{CI}$ & Mean & $95 \% \mathrm{CI}$ & \\
\hline \multirow[t]{4}{*}{$K^{\text {trans }}\left(* 10^{3 *} \min ^{-1}\right)$} & Tumor & 14.6 & $14.1-15.1$ & 3.8 & $3.6-3.9$ & $<0.0001$ \\
\hline & $\operatorname{Rim}$ & 26.3 & $24.5-28.1$ & 4.5 & $4.2-4.9$ & $<0.0001$ \\
\hline & Core & 5.4 & $4.6-6.1$ & 1.8 & $1.5-2.1$ & $<0.0001$ \\
\hline & Muscle & 3.3 & $1.7-4.7$ & 2.1 & $1.9-2.3$ & 0.12 \\
\hline \multirow[t]{4}{*}{$V_{e}$} & Tumor & 20.3 & $19.1-21.5$ & 2.4 & $1.9-2.8$ & $<0.0001$ \\
\hline & $\operatorname{Rim}$ & 32.7 & $29.2-36.1$ & 5.8 & $4.3-7.3$ & $<0.0001$ \\
\hline & Core & 6.3 & $3.8-8.8$ & 0.5 & $0-1.1$ & $<0.0001$ \\
\hline & Muscle & 1.2 & $0.3-2.1$ & 0.8 & $0.3-1.3$ & 0.41 \\
\hline
\end{tabular}


Table 2. Comparison of tissue $\mathrm{pO}_{2}$ in the tumor rim and core before and after fractionated radiotherapy

\begin{tabular}{lccccccc}
\hline & \multicolumn{6}{c}{ Tissue $\mathrm{pO}_{2}(\mathrm{~mm} \mathrm{Hg})$} \\
\cline { 2 - 3 } & \multicolumn{2}{c}{$\begin{array}{c}\text { Before } \\
\text { radiotherapy }\end{array}$} & & \multicolumn{2}{c}{$\begin{array}{c}\text { After } \\
\text { radiotherapy }\end{array}$} \\
\cline { 2 - 3 } & Mean & $95 \%$ CI & & Mean & $95 \%$ CI & $p$ \\
\hline Tumor rim & 6.8 & $6.7-6.8$ & & 7.7 & $7.6-7.8$ & $<0.001$ \\
Tumor core & 3.5 & $3.4-3.5$ & & 4.4 & $4.3-4.4$ & $<0.001$ \\
$p$ & $<0.001$ & & $<0.001$ & & \\
\hline
\end{tabular}

Fig. 4. Both before and after RT, the tumor core was significantly more hypoxic compared with the tumor rim. Fractionated RT significantly increased mean $\mathrm{pO}_{2}$ in both tumor core and rim. The hypoxic fraction $\left(\mathrm{pO}_{2}<5 \mathrm{~mm} \mathrm{Hg}\right)$ in the tumor core was $79.2 \%$ before and $61.9 \%$ after RT $(p=0.008)$. In the tumor rim, the hypoxic fraction was $36.6 \%$ before RT and $29.9 \%$ after RT $(p=0.3)$.

(a)
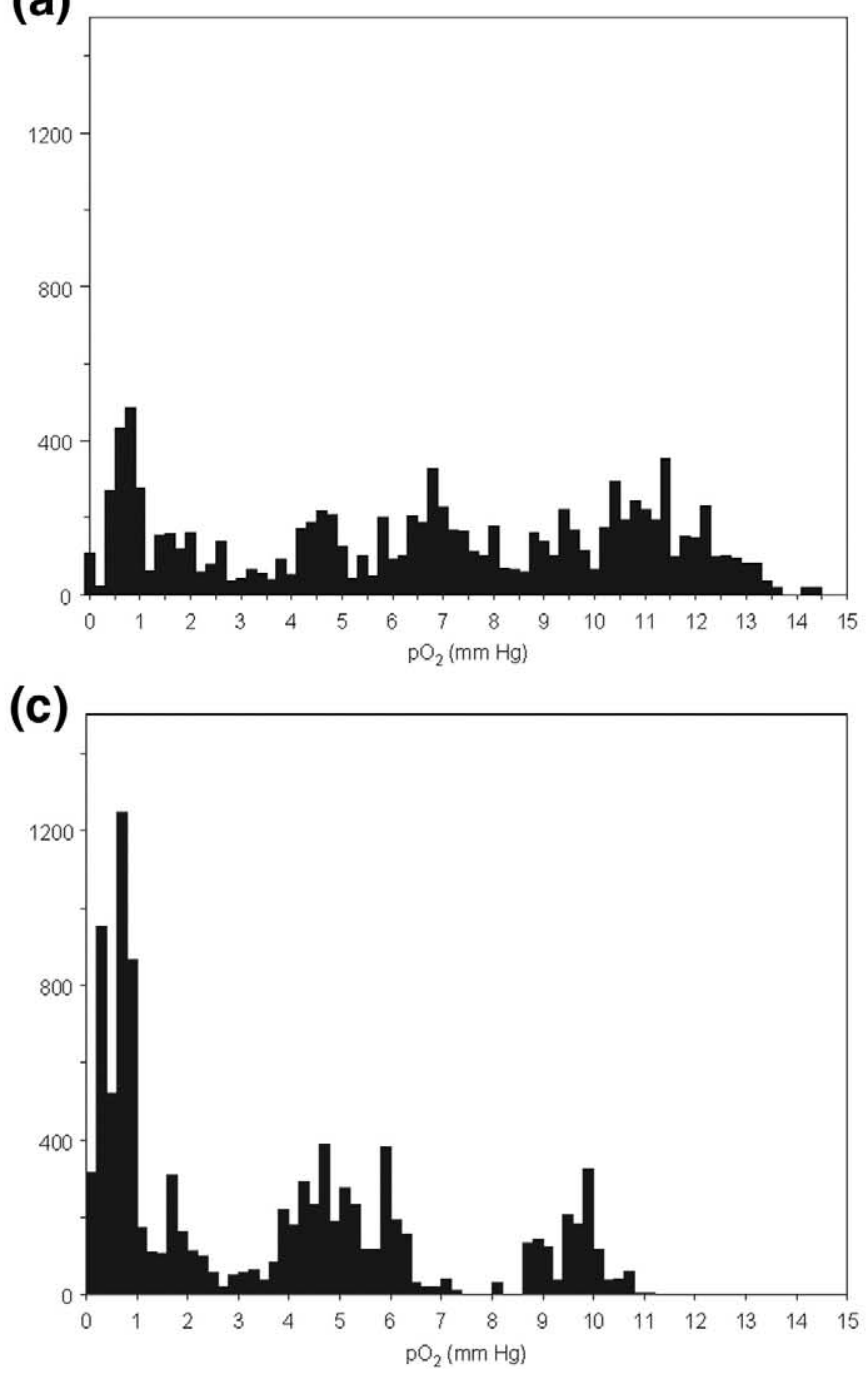

The histogram of cumulative $\mathrm{pO} 2$ readings showed a bimodal distribution in both tumor regions.

Comparison of MVD, pimonidazole staining, and VEGF expression between irradiated and nonirradiated tumor bearing control rats

Expression of VEGF was significantly higher in RT-treated rats in both tumor rim and core. The pimonidazole hypoxia score, however, did not significantly differ between RT-treated and control animals. In the tumor rim, mean MVD was lower in RT-treated animals, but the difference did not reach statistical significance. In the tumor core, MVD was significantly lower compared with the tumor rim, but did not differ between control and RT-treated animals (Table 3).

Statistical correlation of DCE-MRI parameters with $\mathrm{pO}_{2}$ and histologic parameters in the tumor rim after $R T$

After RT, $\mathrm{pO}_{2}$ in the tumor rim was inversely related to $K^{\text {trans }}(r=-0.57, p=0.085)$ and $v_{e}(r=-0.65, p=0.04)$
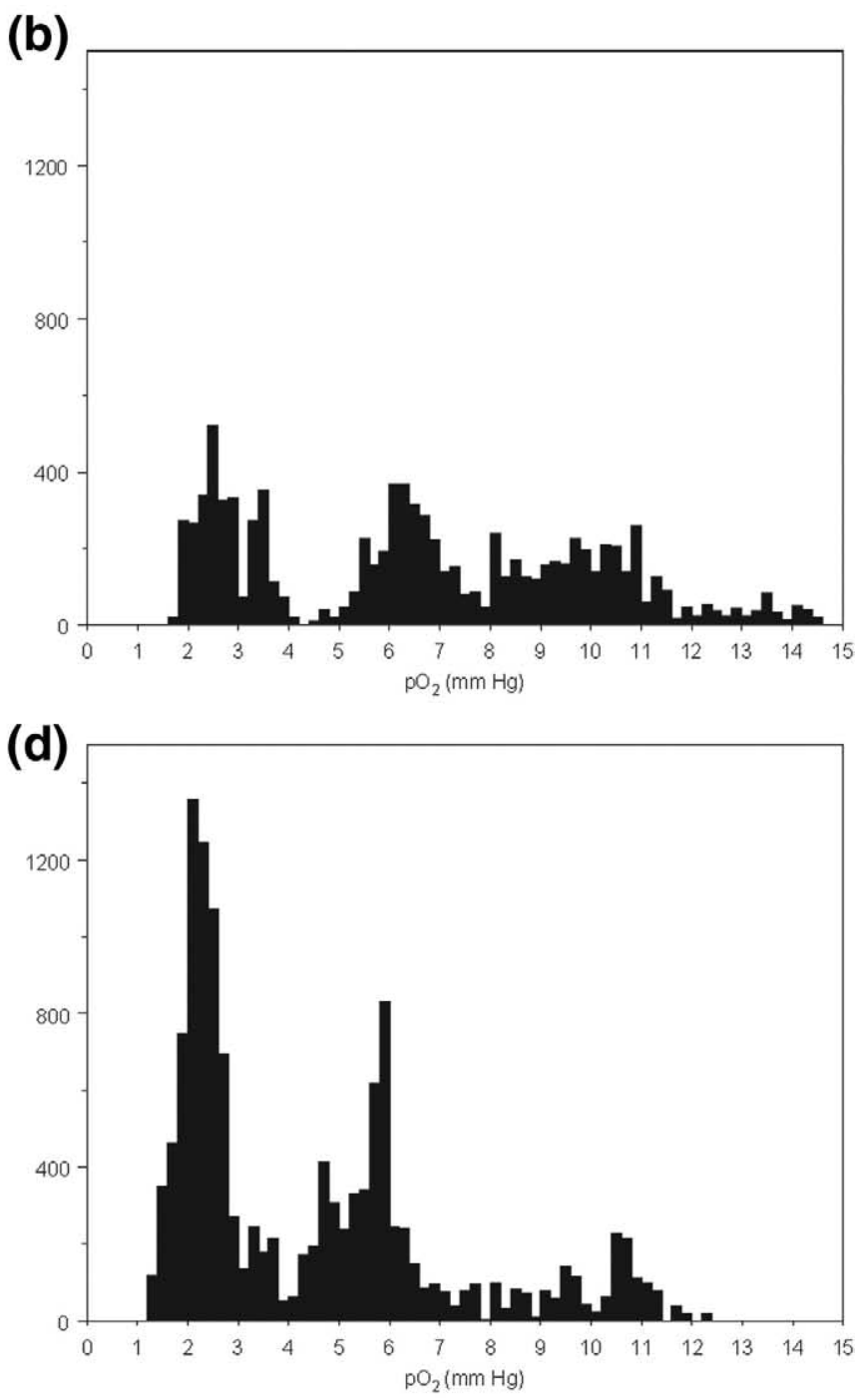

Fig. 4. Frequency histogram of cumulative $\mathrm{pO}_{2}$ readings before radiotherapy (RT) in the tumor rim (a), after $\mathrm{RT}$ in the tumor rim (b), before RT in the tumor core (c), and after RT in the tumor core (d). 
Table 3. Comparison of pimonidazole hypoxia score, microvascular density (MVD), and vascular endothelial growth factor (VEGF) expression between radiotherapy-treated rats and control rats bearing a tumor of similar size

\begin{tabular}{|c|c|c|c|}
\hline & Controls & $\begin{array}{l}\text { Radiotherapy } \\
\text { treated }\end{array}$ & $p$ \\
\hline \multicolumn{4}{|c|}{$\begin{array}{l}\text { VEGF expression score } \\
\text { (median) }\end{array}$} \\
\hline Tumor rim & 2 & 5 & 0.005 \\
\hline Tumor core & 2 & 5 & 0.018 \\
\hline \multicolumn{4}{|c|}{ Hypoxia score (median) } \\
\hline Tumor rim & 4 & 3.5 & 0.33 \\
\hline Tumor core & 4.5 & 4 & 0.79 \\
\hline \multicolumn{4}{|l|}{ MVD (mean) } \\
\hline Tumor rim & 16.9 & 10.4 & 0.061 \\
\hline Tumor core & 5.3 & 6.1 & 0.33 \\
\hline
\end{tabular}

(Fig. 5). No correlation, however, was found between $\mathrm{pO}_{2}$ and signal intensity AUC after RT. Similarly, no significant correlations were found between DCE-MRI parameters and histologic parameters (MVD, VEGF expression, pimonidazole hypoxia score).

\section{DISCUSSION}

Tumor vessels display ultrastructural abnormalities including transcellular openings, widened interendothelial junctions, abnormal endothelial cells, and absent basement membrane that increase permeability even for large molecules (20). In this study, we examined the use of P792, a new macromolecular CA, to measure changes in neovascular leakage induced by fractionated short-term RT. Neovascular leakage is an important physiologic tumor parameter that has been shown to respond quickly and dramatically to antiangiogenesis interventions (21). Leakage of contrast agent is determined both by endothelial permeability and by the total exchange surface area (proportional to the number of functional vessels). Microvessel counts were performed to differentiate permeability effects of RT from changes in the total surface area. To optimize imaging parameters describing the contrast agent dynamics, a high temporal resolution scanning method was used with contrast administered as a rapid central venous bolus (22). DCE-MRI was compared with invasive $\mathrm{pO}_{2}$ mapping. The fluorescence lifetime method we have used to measure $\mathrm{pO} 2$ has several advantages over the Eppendorf device, including absence of oxygen consumption and increased accuracy at low oxygen tensions common in neoplastic tissue (23). We chose to study post-RT effects relatively early to reflect clinical practice, with surgery after short term RT being usually performed within 1 week after the end of therapy.

Our main finding is a significant reduction of $K^{\text {trans }}$ after short-term fractionated RT. Although the MVD did not differ significantly between RT-treated and control animals, a trend toward lower MVD in irradiated rats was present. Although the present analysis of a small number of animals does not allow us to draw a definitive conclusion, probably both altered endothelial permeability and a change in total microvascular surface area contribute to the decreased $K^{\text {trans }}$ after RT. Expression of VEGF was significantly higher in RT-treated rats compared with control animals. Hypoxia as a trigger of VEGF expression was decreased after RT in this model. However, other radiation-induced activators of VEGF expression such as the mitogen activated protein kinase pathway have been shown to enhance VEGF expression after RT (24). Overexpression of VEGF after RT has also been clinically demonstrated in rectal cancer patients (25).

Published experimental data suggest that, depending on the dose and fractionation of RT and the timing of permeability measurement, neovascular permeability can be either increased or decreased (26). Large, single RT doses disrupt the endothelial lining and cause a short-term increase in endothelial permeability. Smaller or fractionated doses, however, tend to decrease vascular permeability or cause no
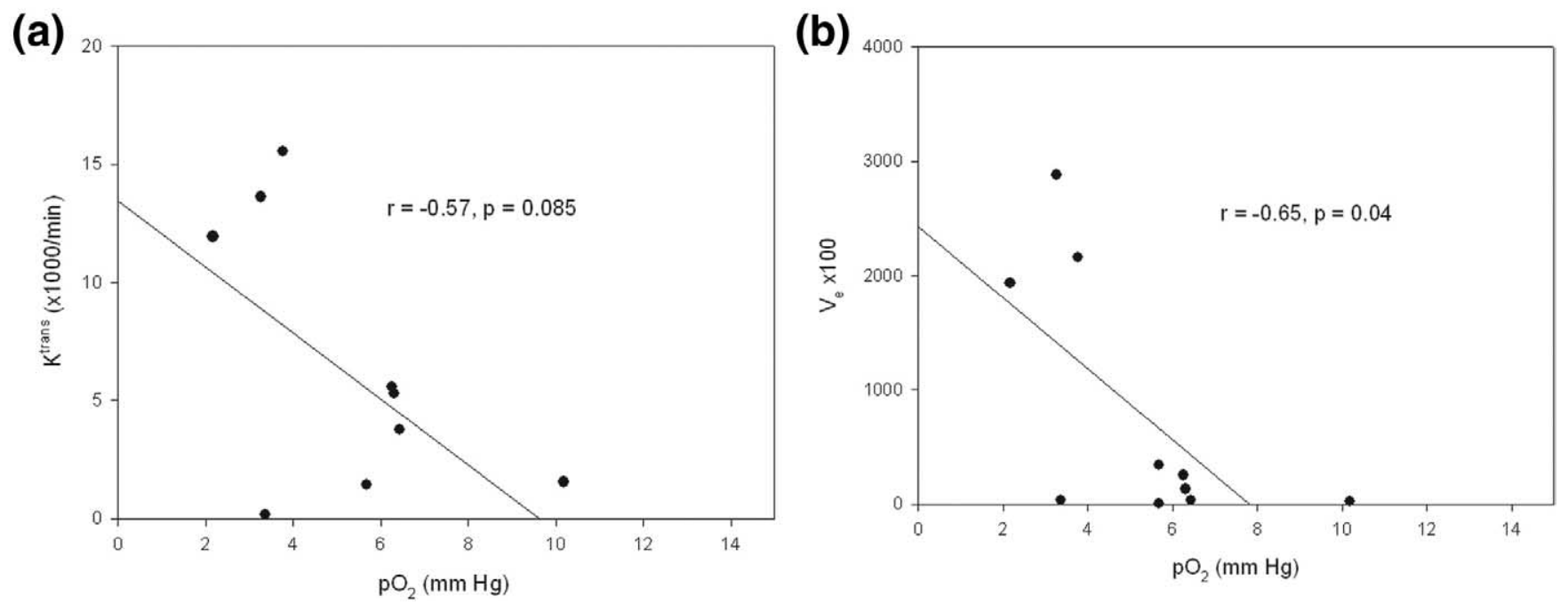

Fig. 5. Correlation of $K^{\text {trans }}$ (a) and $V_{e}$ (b) with oxygenation after radiotherapy. 
change in this parameter. Several authors have used DCEMRI to study the effects of RT on microvascular physiology. Yu et al. used DCE-MRI with gadopentetate dimeglumine (Gd-DTPA) and the macromolecular CA gadomer-17 to study microvascular permeability in a rat adenocarcinoma model (27). They observed a $67 \%$ decrease in permeability measured using gadomer-17 at 3 days after a single dose of $5 \mathrm{~Gy}$, whereas no change was observed after a dose of $20 \mathrm{~Gy}$. No changes in permeability were observed with either dose of RT when Gd-DTPA was used as a contrast agent. Kobayashi et al. studied neovascular permeability with a macromolecular CA after a single dose of RT or fractionated RT (28). They found an increased permeability after a single dose of $15 \mathrm{~Gy}$, whereas permeability was unaffected by fractionated RT.

Several clinical studies have used DCE-MRI with small molecular weight MRI contrast agents to study tumor microenvironment during rectal cancer therapy. George et al. found a significant relation between pretreatment $K^{\text {trans }}$ assessed with Gd-DTPA and response to neoadjuvant chemoradiation (29). Responsive tumors showed a marked reduction in $K^{\text {trans }}$ at the end of treatment (mean logarithmic $K^{\text {trans }}-0.46$ vs. $0.86 ; p=0.04$ ). It should be noted that with the use of Gd-DTPA, $K^{\text {trans }}$ probably represents both permeability and microvascular flow. Gd-DTPA as a contrast agent was also used by de Vries et al., who monitored microcirculation during chemoradiation for rectal cancer (30). They calculated a perfusion index from the shape of the arterial and tumor tissue curves and found this value to be significantly increased up to 2 weeks after the start of chemoradiation. In patients treated with surgery only, Tuncbilek et al. found a significant relation between pretreatment descriptive DCE-MRI parameters and microvessel density, tumor grade, and patient outcome (31).

We found that the biophysical and imaging properties of P792 allow investigators to selectively study neoplastic vascular physiology and to monitor the effects of RT on tumor microvasculature. Several authors have used P792 to characterize tumor physiology in animal models. Turetschek et al. found no relation between transendothelial permeability estimated with P792 and histologic parameters (microvessel density and tumor grade) in a breast cancer model (32). In a prostate cancer model, however, P792-based DCE-MRI was successfully used to monitor changes in permeability after anti-VEGF therapy (15). It is likely, therefore, that successful estimation of neovascular permeability with P792 is highly dependent not only on imaging and image processing methodology, but also on tumor type and grade. A comparison of gadopentetate dimeglumine and P792 to characterize tumor physiology and metastatic ability in a rodent prostatic cancer was published by Fan et al. (33). They used an empiric mathematical model to fit the observed enhancement curves. In agreement with our findings, uptake of P792 was slow and influenced primarily by capillary leakage, with a strong uptake difference between tumor and normal tissue.

We combined DCE-MRI with invasive $\mathrm{pO}_{2}$ measurements and found a significant increase in $\mathrm{pO}_{2}$ in the peripheral region of the tumor that was sampled with a fiberoptic probe. The available evidence concerning the effect of fractionated radiation on tumor oxygenation suggests that either an increase or a decrease in oxygenation can be observed depending on RT dose, fractionation, tumor histology, and timing of $\mathrm{pO}_{2}$ measurement (34). Increased oxygenation shortly after fractionated radiotherapy has been previously reported and is the net result of changes in both oxygen supply (reduced interstitial pressure, increased flow) and oxygen consumption (decreased cell density) (35). Our results suggest a bimodal distribution of tumor $\mathrm{pO}_{2}$ values, a finding previously reported in a prostate cancer model using a noninvasive assay (36). Oxygenation was sampled over a 4-mm trajectory; the observed bimodal distribution therefore probably represents intralesion heterogeneity with severely hypoxic zones and better oxygenated zones surrounding feeding vessels. Before RT, no relation could be demonstrated between $\mathrm{pO}_{2}$ measurements and DCE-MRI parameters in the tumor rim. After RT, however, high $\mathrm{pO}_{2}$ values corresponded with low $K^{\text {trans }}$ and $v_{e}$ values, whereas no relation was found with the signal intensity AUC. This finding probably reflects the stabilizing effect of RT on tumor vasculature with a partial return of the physiologic relationship between well-oxygenated tissue and a structurally normal microvessel wall. Moreover, our data support the use of pharmacokinetic modeling of dynamic MRI data to generate parameters which directly reflect physiologic processes in contrast with mere description of the tissue enhancement curve.

In conclusion, the biophysical properties of P792 allow noninvasive measurement of microvascular leakage in a colorectal tumor model. Fractionated RT markedly decreases contrast leakage by altered neovascular permeability. After RT, reoxygenation of tumor tissue corresponds with a lowered transfer constant and leakage space. DCEMRI could therefore be a tool in noninvasive monitoring of tumor microvascular response to fractionated RT. Future work in this model will combine RT with chemotherapy and targeted therapy.

\section{REFERENCES}

1. Bray F, Sankila R, Ferlay J, Parkin DM. Estimates of cancer incidence and mortality in Europe in 1995. Eur J Cancer 2002;38:99-166.

2. Sauer R, Becker H, Hohenberger W, et al. Preoperative versus postoperative chemoradiotherapy for rectal cancer. New Engl J Med 2004;351:1731-1740.

3. Camma C, Giunta M, Fiorica F, et al. Preoperative radiother- apy for resectable rectal cancer-a meta-analysis. JAMA 2000;284:1008-1015.

4. King M, Tolan S, Giridharan S, et al. Late toxicity after short course preoperative radiotherapy and total mesorectal excision for resectable rectal cancer. Clin Oncol 2003;15: 233-236.

5. Beets-Tan RGH, Vliegen RFA, Beets GL. Magnetic reso- 
nance imaging of rectal cancer: What radiation oncologists need to know. Front Radiat Ther Oncol 2004;38:1-12.

6. Padhani AR. Dynamic contrast-enhanced MRI in clinical oncology: Current status and future directions. J Magn Reson Imaging 2002;16:407-422.

7. Tofts PS. Modeling tracer kinetics in dynamic Gd-DTPA MR imaging. J Magn Reson Imaging 1997;7:91-101.

8. Turetschek K, Floyd E, Helbich T, et al. MRI assessment of microvascular characteristics in experimental breast tumors using a new blood pool contrast agent (MS-325) with correlations to histopathology. J Magn Reson Imaging 2001;14: 237-242.

9. Turetschek K, Preda A, Floyd E, et al. MRI monitoring of tumor response following angiogenesis inhibition in an experimental human breast cancer model. Eur J Nucl Med Mol Imaging 2003;30:448-455.

10. Fournier SL. The role of blood pool contrast media in the study of tumor pathophysiology. In: Baert AL, Sartor K, editors. Dynamic contrast-enhanced magnetic resonance imaging in oncology. Berlin: Springer; 2005. p. 39-52.

11. Hagenaars M, Koelemij R, Ensink NG, et al. The development of novel mouse monoclonal antibodies against the CC531 rat colon adenocarcinoma. Clin Exp Metastas 2000; 18:281-289.

12. Ceelen W, El Malt M, Cardon A, et al. Influence of preoperative high-dose radiotherapy on postoperative outcome and colonic anastomotic healing-experimental study in the rat. Dis Colon Rectum 2001;44:717-721.

13. El-Malt M, Ceelen W, De Meerleer G, et al. Influence of preoperative combined radiochemotherapy on surgical outcome and colonic anastomotic healing: Experimental study in the rat. Int J Radiat Oncol 2001;50:1073-1078.

14. Port M, Corot C, Rousseaux O, et al. P792: A rapid clearance blood pool agent for magnetic resonance imaging: Preliminary results. Magn Reson Mater Phy 2001;12:121-127.

15. Pradel C, Siauve N, Bruneteau G, et al. Reduced capillary perfusion and permeability in human tumour xenografts treated with the VEGF signalling inhibitor ZD4190: An in vivo assessment using dynamic MR imaging and macromolecular contrast media. Magn Reson Imaging 2003;21:845-851.

16. Brurberg KG, Graff BA, Olsen DR, et al. Tumor-line specific $\mathrm{pO}(2)$ fluctuations in human melanoma xenografts. Int $J$ Radiat Oncol 2004;58:403-409.

17. Blackwell KL, Kirkpatrick JP, Snyder SA, et al. Human recombinant erythropoietin significantly improves tumor oxygenation independent of its effects on hemoglobin. Cancer Res 2003;63:6162-6165.

18. Weidner N, Semple JP, Welch WR, et al. Tumor angiogenesis and metastasis-correlation in invasive breast-carcinoma. New Engl J Med 1991;324:1-8.

19. Coppola D, Lu L, Fruehauf JP, et al. Analysis of p53, p21(WAF1), and TGF-beta 1 in human ductal adenocarcinoma of the pancreas-TGF-beta 1 protein expression predicts longer survival. Am J Clin Pathol 1998;110:16-23.

20. Carmeliet P, Jain RK. Angiogenesis in cancer and other diseases. Nature 2000;407:249-257.
21. Brasch R, Turetschek K. MRI characterization of tumors and grading angiogenesis using macromolecular contrast media: Status report. Eur J Radiol 2000;34:148-155.

22. Henderson E, Rutt BK, Lee TY. Temporal sampling requirements for the tracer kinetics modeling of breast disease. Magn Reson Imaging 1998;16:1057-1073.

23. Dewhirst MW, Klitzman B, Braun RD, et al. Review of methods used to study oxygen transport at the microcirculatory level. Int J Cancer 2000;90:237-255.

24. Park JS, Qiao L, Su ZZ, et al. Ionizing radiation modulates vascular endothelial growth factor (VEGF) expression through multiple mitogen activated protein kinase dependent pathways. Oncogene 2001;20:3266-3280.

25. Nozue M, Isaka N, Fukao K. Over-expression of vascular endothelial growth factor after preoperative radiation therapy for rectal cancer. Oncol Rep 2001;8:1247-1249.

26. Baker DG, Krochak RJ. The response of the microvascular system to radiation-a review. Cancer Invest 1989;7:287-294.

27. $\mathrm{Yu} \mathrm{H}, \mathrm{Su} \mathrm{MY}$, Wang ZH, et al. A longitudinal study of radiation-induced changes in tumor vasculature by contrastenhanced magnetic resonance imaging. Radiat Res 2002;158: 152-158.

28. Kobayashi H, Reijnders K, English S, et al. Application of a macromolecular contrast agent for detection of alterations of tumor vessel permeability induced by radiation. Clin Cancer Res 2004;10:7712-7720.

29. George ML, Dzik-Jurasz ASK, Padhani AR, et al. Non-invasive methods of assessing angiogenesis and their value in predicting response to treatment in colorectal cancer. $\mathrm{Br}$ J Surg 2001;88:1628-1636.

30. de Vries A, Griebel J, Kremser C, et al. Monitoring of tumor microcirculation during fractionated radiation therapy in patients with rectal carcinoma: Preliminary results and implications for therapy. Radiology 2000;217:385-391.

31. Tuncbilek N, Karakas HM, Altaner S. Dynamic MRI in indirect estimation of microvessel density, histologic grade, and prognosis in colorectal adenocarcinomas. Abd Imaging 2004; 29:166-172.

32. Turetschek K, Floyd E, Shames DM, et al. Assessment of a rapid clearance blood pool MR contrast medium (P792) for assays of microvascular characteristics in experimental breast tumors with correlations to histopathology. Magn Reson Med 2001;45:880-886.

33. Fan XB, Medved M, River JN, et al. New model for analysis of dynamic contrast-enhanced MRI data distinguishes metastatic from nonmetastatic transplanted rodent prostate tumors. Magn Reson Med 2004;51:487-494.

34. Molls M. Changes in tumor oxygenation during radiation therapy. In: Molls M, Vaupel P, editors. Blood perfusion and microenvironment of human tumors. Implications for clinical radiooncology. Berlin: Springer; 2000. p. 81-88.

35. Stuben G, Thews O, Pottgen C, et al. Tumour oxygenation during fractionated radiotherapy-comparison with sizematched controls. Acta Oncol 1999;38:209-213.

36. Mason RP, Hunjan S, Le D, et al. Regional tumor oxygen tension: Fluorine echo planar imaging of hexafluorobenzene reveals heterogeneity of dynamics. Int J Radiat Oncol 1998; 42:747-750. 